\title{
Lymph Nodes Invasion of Marcille's Fossa Associates with High Metastatic Load in Prostate Cancer Patients Undergoing Extended Pelvic Lymph Node Dissection: The Role of "Marcillectomy"
}

\author{
Antonio B. Porcaro ${ }^{a}$ Giovanni E. Cacciamani ${ }^{a}$ Marco Sebben ${ }^{a} \quad$ Alessandro Tafuri $^{a}$ \\ Tania Processalia Riccardo Rizzetto ${ }^{a}$ Nicolò De Luyk ${ }^{a}$ Marco Pirozzi ${ }^{a}$ \\ Nelia Amigoni ${ }^{a}$ Paolo Corsi ${ }^{a}$ Davide Inverardi ${ }^{a}$ Matteo Brunelli ${ }^{b}$ \\ Filippo Migliorini $^{\mathrm{a}}$ Vincenzo De Marco ${ }^{\mathrm{a}}$ Walter Artibani ${ }^{\mathrm{a}}$ \\ ${ }^{a}$ Department of Urology, University of Verona, Verona, Italy; ${ }^{b}$ Department of Pathology, University of Verona, Verona, Italy
}

\section{Keywords}

Marcilles's fossa - Marcille's triangle · Prostate cancer ·

Extended pelvic lymph node dissection

\begin{abstract}
Introduction: To assess the incidence of malignancy involvement of lymph nodes (LNs) in Marcille's fossa in patients undergoing robot assisted radical prostatectomy (RARP) and extended pelvic lymph nodes dissection (ePLND) for prostate cancer (PCa). Design, Setting, and Participants: Between January 2014 and December 2017, details of patients who underwent RARP and ePLND were prospectively analysed. All the nodal packets were dissected separately, grouped into left and right nodes and submitted in separate packages to dedicated pathologist. Results and Limitations: Two hundred and twenty-one patients underwent ePLND and RARP in the study period. In aggregate, Marcille's LNs involvement was found in 5 (2.3\%) of patients, 2 on the left side and 3 on the right side. Per cent of positive cores and Gleason at biopsy are clinical predictors of LNs invasion; moreover, in the surgical specimen, seminal vesicle invasion and high-grade cancer were factors related to loco-regional
\end{abstract}

metastases. Conclusions: Marcille's nodes involvement is associated to contemporarily multiple LN metastases in other template locations in high-risk PCa patients. The Marcille's lymphadenectomy would be recommended when planning an ePLND in high-risk PCa.

(c) 2019 S. Karger AG, Basel

\section{Introduction}

Prostate cancer $(\mathrm{PCa})$ can disseminate through lymphatics, peri-neural spaces and venous routes. Obviously, this condition influences its natural history. Periprostatic lymphatic networks include ascending, lateral and posterior routes [1].

Nowadays, both conventional and functional imaging have poor accuracy in detecting preoperative nodal status, because of many early metastases of lymph nodes (LN) in $\mathrm{PCa}$ are micro or occult metastases [2]. Extended pelvic lymph nodes dissection (ePLND) results to be the most

A.B.P. and G.E.C. contribute equally to the paper. 

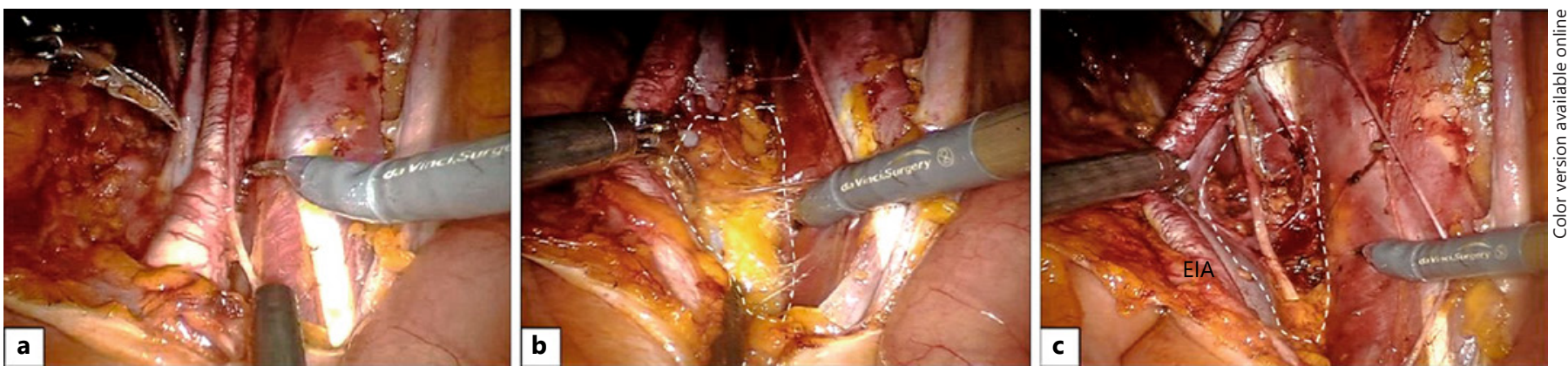

Fig. 1. Steps of the Marcillectomy during ePLND for PCa. The dotted-dash line area indicates the exposed Marcille's fossa (a) identification of the external iliac LN group which is dissected laterally to the genitofemoral nerve at the lateral edge of the internal iliac artery and vein. $\mathbf{b}$ With the Prograsp ${ }^{\mathrm{TM}}$ the external iliac vessels are pulled medially permitting lymphatic removal posterior to these vessels. $\mathbf{c}$ This enables the visualization posteriorly to the obturator nerve, at the level of the pelvic floor, when the triangle of Marcille's and deep LN packets are removed. Steps of the Marcillectomy during ePLND for PCa. The dotted-dash line area indicates the exposed Marcille's fossa. accurate nodal staging procedure in PCa [3]. European Urology Association guidelines recommend performing extended ePLND in intermediate [4] and high-risk PCa patients [5]. Multiple statistical models predicting lymph node involvement in PCa exist to support clinical decision-making regarding extended pelvic ePLND [6].

However, there is still no agreement on the lymph nodal fields that should be removed and different ePLND templates have been proposed [7-9].

A progressive extension of the anatomical template by adding more nodal fields would further reduce the risk of missing positive LN; however, it may lead to longer operating times and a higher risk of complications [10].

The Marcille's triangle or fossa is a pelvic anatomical region, which is limited by the fifth lumbar vertebra medially, from the inner edge of the muscle large psoas laterally, from the upper edge of the wing of the sacrum below $[11,12]$. LNS of this anatomical are related to the prostate lymphatic system [9]. However, the dissection of Marcille's LNs is a subject that has not been investigated in robotic surgery.

The aim of the present study is to assess both the prevalence and the incidence of malignancy involvement of nodes in Marcille's Triangle in patients undergoing robot assisted radical prostatectomy (RARP) and ePLND for PCa.

\section{Methods}

Between January 2014 and December 2017, details of all consecutive patients who underwent RARP and ePLND with intent to cure locally advanced PCa performed by 2 expert surgeons (W.A. and V.D.M.) were identified from our prospectively collected in an
Institutional database. All the procedures were recorded, and video-tapes were examined at any convenience. Patients who underwent androgen deprivation therapy before the surgery were excluded from the analysis. Pre-operative data collected included age (years), body max index $\left(\mathrm{kg} / \mathrm{m}^{2}\right)$, baseline total prostate specific antigen $(\mathrm{ng} / \mathrm{mL})$ serum levels, percentage of biopsy positive cores $(\%)$, total prostate volume $(\mathrm{mL})$, tumour and nodal clinical stage and Gleason Grading Group in biopsy cores. Postoperative complication rates, classified according to Clavien-Dindo classification, were also assessed [13]. Patients were classified using the D'Amico clinical class risk categories [14] and staged according to the 2010 American Joint Committee on Cancer staging system for PCa.

The decision to perform an extended LN dissection was mainly based on pre-operative nomograms showing a risk of LN invasion greater than $5 \%$ [15]. In low-risk patients, the decision to perform an ePLND was based on clinical factors indicating an increased risk of tumour upgrading in the surgical specimen [16-19]. Dissected pelvic LNs were sampled according to a standardized anatomical template as follows: external iliac, Cloquet's, Obturator, Marcille's, and internal iliac nodes.

The Marcille's fossa or Marcille's Triangle is an anatomical region located in the vicinity of the lateral part of the fifth lumbar vertebra, the medial border of the psoas muscle laterally and the cephalad surface of the ala of the sacrum [11]. Figure 1 shows the surgical steps of the LNs dissection located in the Marcille's fossa: the vas deferens is transected along its course. The external iliac $\mathrm{LN}$ group is dissected laterally to the genitofemoral nerve at the lateral edge of the internal iliac artery and vein from the node of Cloquet to the ureteric crossing of the internal iliac artery. With the Prograsp ${ }^{\mathrm{TM}}$ at the 4 th arm, the external iliac vessels are pulled medially permitting lymphatic removal posterior to these vessels. This enables the visualization posteriorly to the obturator nerve, at the level of the pelvic floor, when the triangle of Marcille's and deep LN packets are removed.

All the nodal packets were dissected sequentially and separately and then taken out through the $12-\mathrm{mm}$ trocar. Surgical specimens were evaluated by the dedicated pathologist. Nodal packets 
were grouped into left and right nodes, tagged and submitted in separate packages according to the Stanford protocol $[5,20]$. Dissected LNs were counted and evaluated for cancer involvement, which was classified as absent, $<5$ or $\geq 5$ nodes. All specimens were processed separately. Tumours were classified according to the Gleason Grading Group system [21].

The removed LNs were assessed for histopathological analysis. Pathologic work-up to detect LNs in the specimens comprised direct visualization, palpation and standard hematoxylin-eosin staining. Immunohistochemical staining was performed when appropriate. In each case, the numbers of removed and metastatic nodes were assessed. Prostate and nodal specimens were then staged according to the 2010 American Joint Committee on Cancer staging system for PCa.

\section{Statistical Analysis}

The patient's population was grouped according to LNs invasion (present or absent). Metastatic cases were classified according to the presence or absence of involvement or Marcille's fossa.

A descriptive analysis was performed reporting continuous and categorical variables as median, interquartile range (IQR) and frequencies, percentages (\%). In the univariate analysis KruskalWallis, chi-square and Fisher exact tests were used to compare continuous and categorical variables as appropriate.

A 2-tailed test with $p<0.05$ was considered statistically significant. All statistical analyses were performed using SPSS version24.0 (SPSS Inc., Chicago, IL, USA).

\section{Results}

Between January 2013 and March 2017, a total of 221 patients underwent RARP and ePLND in our Institution including Marcille's fossa dissection bilaterally. Demographical and pathological data of the study populations are displayed in Table 1. Of the 221 investigated subjects, 30 (13.5\%) had ASA score I, 175 (79.2\%) ASA score II, and $15(7.2 \%)$ ASA score III. The overall median (and IQR) baseline age, body max index, prostate volume, proportion of positive cores, number of dissected LN, operating time, blood loss, and length of hospital stay are reported. Of the 221 patients included in the study, 26 (11.8\%) were low risk, 129 (58.4\%) intermediate risk, and 66 (29.9\%) high risk. A total of 13 (5.9\%) cases had clinical extraprostatic extension of the tumour. Suspected clinical LN invasion (enlarged nodes) was observed in 10 (4.5\%) patients. A total of 43 (19.4\%) patients presented with a Gleason Grading Group $\geq 4$ at biopsy. In the surgical specimen, Gleason Grading Group score $\geq 4$ was detected in $71(32.2 \%)$ cases. Extraprostatic extension of cancer was observed in $68(30.7 \%)$ patients of whom 29 (13.1\%) had extracapsular extension and $39(17.6 \%)$ seminal vesicle invasion. A total of $62(28.1 \%)$ subjects had surgical margins invaded by cancer. No port-site recur- rence on the abdominal wall was reported. Symptomatic lymphocele was observed in 5 (1.9\%) subjects of whom 2 underwent percutaneous drainage and surgical drainage.

All the specimens were examined by dedicated pathologist for evaluation. A median (IQR) of 28 (22-35) LNs were yielded. Globally $30(13.5 \%)$ patients presented with an LNs invasion.

Figure 2 depicts the distribution of nodes removed from Marcille's fossa. No LN was found in 28.5 and $19.9 \%$ of cases in right and left triangle; In the 68.8 and $75.9 \%$ of cases a Marcille's count $<5$ nodes was found on the left and right side, while $\geq 5$ nodes was found in 2.7 and $4.2 \%$ in the left and right Marcille's Triangle respectively. In aggregate, Marcille's LNs involvement was found in 5 (2.3\%) of patients, 2 on the left side and 3 on the right side. No patient presented with the bilateral involvement of Marcille's fossa.

Table 2 depicts demographic, surgical and pathological data of the population with positive nodes. No differences were found when comparing patients with positive vs. negative Marcille's nodes except for the total number (median, IQR) of LNs with metastatic involvement that was found higher in patients with Marcille's node PCa metastasis ( 1 vs. 7 ; OR 2.871, 95\% CI 1,158-7,119; $p=$ $0.023)$.

Table 3 depicts clinical and pathological data of patients with Marcille's node involvement. Four out of 5 patients reported familiarity for PCa and were classified as "high risk" according to D' Amico's classification and presented an extracapsular extension. In all cases, the Marcille's nodes involvement was associated with a metastatic spread in the other lymphatic landing site.

\section{Discussion}

In order to reach the primary goal of oncologic surgery (i.e., complete resection) in pelvic malignancy, LN dissection templates proposed so far were increasingly extended [22]. We have previously demonstrated the role of Cloquet's nodes dissection during RARP and ePNLD [23]. Moreover, the interest on Marcille's region is not exclusive of academic purpose, but it is also important to understand a complex anatomical area in order to perform a lateral pelvic surgery [11]. Seventy-five per cent of all anatomical landing sites were included in the standard extended PLND [24]. Extending the anatomical template by adding more nodal areas could increase LNs removed [25], the chance to detect LN invasion [26] improved survival in patients pN0 [27] (virtually due to the occult micro-metastases removal). 
Table 1. Clinical and pathological factors associated with lymph node invasion in the patient population

\begin{tabular}{|c|c|c|c|c|}
\hline & \multirow[t]{2}{*}{ Population } & \multicolumn{2}{|c|}{ Lymph node invasion } & \multirow[t]{2}{*}{$p$ value } \\
\hline & & absent & present & \\
\hline Age, years, median (IQR) & $66(65-70)$ & $65(61-70)$ & $67(62.7-70.2)$ & 0.189 \\
\hline BMI, kg/m², median (IQR) & $25.3(23.7-27.8)$ & $25.6(23.5-27.8)$ & $25(24.3-27.5)$ & 0.565 \\
\hline PSA, ng/mL, median (IQR) & $6.8(5.1-8.9)$ & $6.8(5.2-8.7)$ & $7.1(4.9-10.1)$ & 0.637 \\
\hline PW, g, median (IQR) & $52(41.5-65)$ & $52(40-65)$ & $55.5(43-68.5)$ & 0.276 \\
\hline LNR, $n(\%)$, median (IQR) & $28(22-34)$ & $28(22-33)$ & $28(23-36.5)$ & 0.628 \\
\hline OT, min, median (IQR) & $225(200-255)$ & $223(200-253)$ & $242.5(200-270.5)$ & 0.255 \\
\hline BL, mL, median (IQR) & $250(150-400)$ & $250(150-400)$ & $250(200-462.5)$ & 0.460 \\
\hline DD, days, median (IQR) & $4(4-5)$ & $4(4-5)$ & $4(4-4.2)$ & 0.366 \\
\hline cN1, $n(\%)$ & $10(4.5)$ & $8(4.2)$ & $2(6.7)$ & \\
\hline BGG $1, n(\%)$ & $46(20.8)$ & $42(22)$ & $4(13.3)$ & 0.002 \\
\hline BGG $2-3, n(\%)$ & $131(59.8)$ & $119(62.3)$ & $13(43.3)$ & \\
\hline BGG $4-5, n(\%)$ & $43(19.4)$ & $28(15.7)$ & $13(43.3)$ & \\
\hline Low-risk class, $n(\%)$ & $26(11.8)$ & $24(12.6)$ & $2(6.7)$ & 0.088 \\
\hline Intermediate-risk class, $n(\%)$ & $129(58.4)$ & $115(60.2)$ & $14(46.7)$ & \\
\hline High-risk class, $n(\%)$ & $66(29.9)$ & $52(27.2)$ & $14(46.7)$ & \\
\hline $\mathrm{pT} 2, n(\%)$ & $153(69.2)$ & $144(75.4)$ & $9(8.30)$ & $<0.0001$ \\
\hline pT3a, $n(\%)$ & $29(13.1)$ & $27(14.1)$ & $2(6.7)$ & \\
\hline pT3b, $n(\%)$ & $39(17.6)$ & $20(810.5)$ & $19(63.3)$ & \\
\hline NSM, $n(\%)$ & $159(71.9)$ & $142(74.3)$ & $17(56.7)$ & 0.045 \\
\hline CDS $3 a-3 b, n(\%)$ & $8(3.6)$ & $6(3.2)$ & $2(6.7)$ & \\
\hline No RAD, $n(\%)$ & $213(96.4)$ & $184(96.3)$ & $29(96.7)$ & 0.928 \\
\hline $\mathrm{RAD}, n(\%)$ & $8(3.6)$ & $7(3.7)$ & $1(3.3)$ & \\
\hline
\end{tabular}

IQR, interquartile range; BMI, body mass index; PSA, prostate specific antigen; TPV, total prostate volume; BPC, biopsy positive cores; PW, prostate weight; LNR, lymph nodes removed; OT, operating time; DD, discharge day; cT, tumour clinical stage; cN, nodal; BGG, biopsy grade group; pT, tumour pathological stage; NSM, negative surgical. margin; PSM, positive surgical margin; PGG, pathological tumour grade group; ASA, American Society of anaesthesiology score system; CDS, Clavien-Dindo score system; RAD, hospital re-admission.

Although the Marcille's Triangle was reported for the first time at the beginning of XIX century [12], fewer and sparse publications described its role in terms of pathological staging and oncological adequacy. A systematic review of the literature was performed in June 2018 by our group using the Medline, Scopus, and Web of Science databases, searching for the terms "Marcille's fossa" or "Marcille's Triangle" in all fields of the records. The systematic search identified 8 studies: 1 anatomical study on cadavers [11], 4 in PCa [9, 28-30], 3 in bladder cancer [31-33]. Only one paper takes into account the "Marcillectomy" as an integral part of the ePLND in 
Fig. 2. Distribution of removed and positive LN in Marcille's Triangle. LNs, lymph nodes.

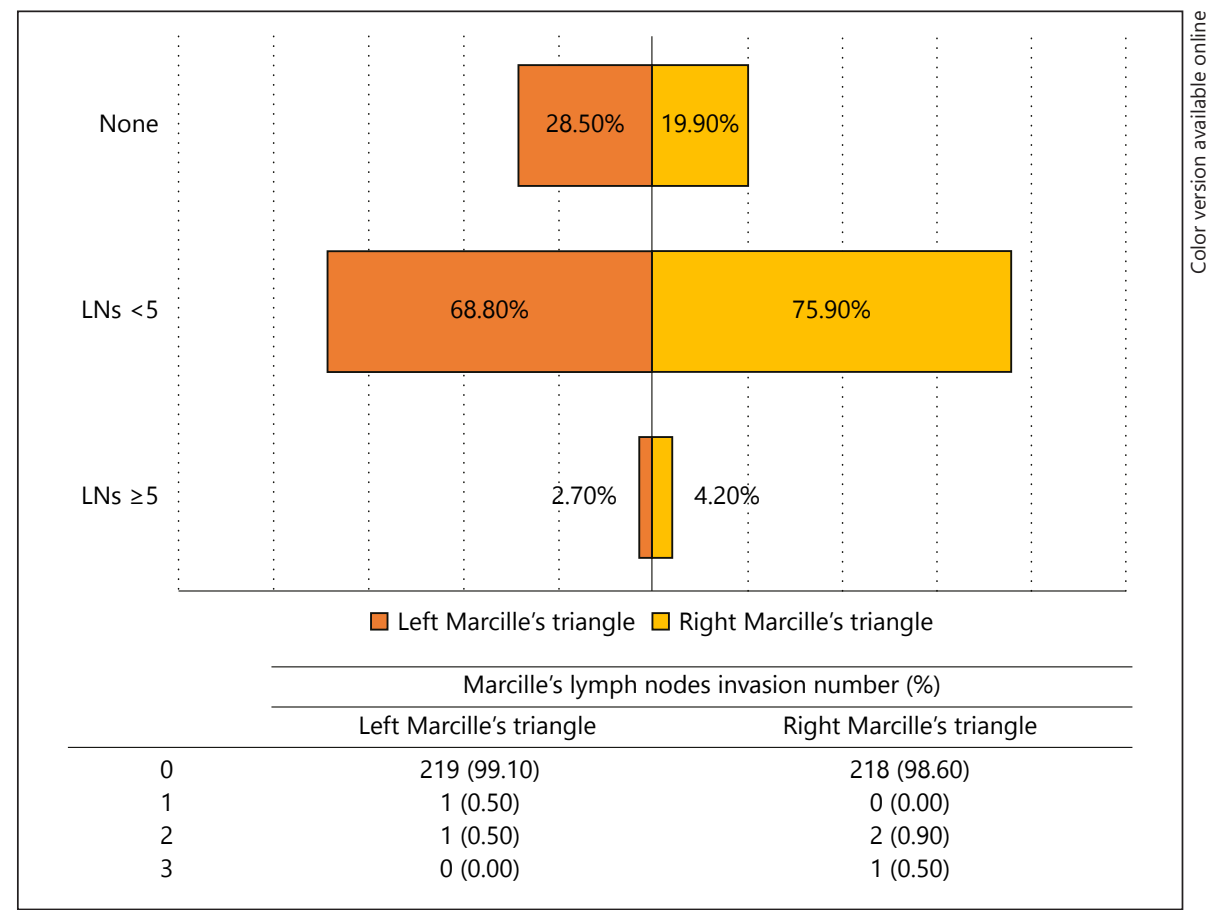

Table 2. Factors associated with involvement of Marcille's Triangle in patients with lymph node metastases

\begin{tabular}{|c|c|c|c|}
\hline & \multicolumn{2}{|c|}{$\begin{array}{l}\text { Lymph node invasion with Marcille's } \\
\text { Triangle involvement }\end{array}$} & \multirow[t]{2}{*}{$p$ value } \\
\hline & absent & present & \\
\hline$n(\%)$ & $25(83.3)$ & $5(16.7)$ & \\
\hline Age, years, median (IQR) & $67(62.5-71.5)$ & $68(64-69.5)$ & 1.000 \\
\hline BMI, $\mathrm{kg} / \mathrm{m}^{2}$, median (IQR) & $25(24.4-27.4)$ & $24.8(22.9-32)$ & 0.957 \\
\hline PSA, ng/mL, median (IQR) & $7.1(4.9-9.8)$ & $8(5.7-16.4)$ & 0.330 \\
\hline $\mathrm{TPV}, \mathrm{mL}$, median (IQR) & $41(28.5-55)$ & $48(35.5-64)$ & 0.388 \\
\hline BPC, $n(\%)$, median (IQR) & $50(38-71)$ & $67(43.5-76.5)$ & 0.435 \\
\hline PW, g, median (IQR) & $54(42-66)$ & $61(51-74)$ & 0.435 \\
\hline LNR, median (IQR) & $28(22.5-35.5)$ & $33(25.5-43.5)$ & 0.253 \\
\hline LNP, median (IQR) & $1(1-2)$ & $7(3.5-15.5)$ & $0.001^{*}$ \\
\hline OT, min, median (IQR) & $234(200-264.5)$ & $257(185-282.5)$ & 0.656 \\
\hline $\mathrm{BL}, \mathrm{mL}$, median (IQR) & $300(200-475)$ & $200(125-675)$ & 0.431 \\
\hline DD, days, median (IQR) & $4(4-4.5)$ & $4(4-9)$ & 0.915 \\
\hline cT1c, $n(\%)$ & $12(48)$ & $2(40)$ & 0.714 \\
\hline $\mathrm{cT} 2, n(\%)$ & $11(44)$ & $2(40)$ & \\
\hline $\mathrm{cT} 3, n(\%)$ & $2(8)$ & $1(20)$ & \\
\hline BGG $1-3, n(\%)$ & $16(64)$ & $1(20)$ & 0.138 \\
\hline BGG $4-5, n(\%)$ & $9(36)$ & $4(80)$ & \\
\hline D'Amico $1-2, n(\%)$ & $15(60)$ & $1(20)$ & 0.157 \\
\hline D'Amico $3, n(\%)$ & $10(40)$ & $4(80)$ & \\
\hline pT $2-3 a, n(\%)$ & $10(40)$ & $1(20)$ & 0.626 \\
\hline pT3b, $n(\%)$ & $15860)$ & $4(80)$ & \\
\hline NSM, $n(\%)$ & $15(60)$ & $2(40)$ & 0.628 \\
\hline PSM, $n(\%)$ & $5(40)$ & $3(60)$ & \\
\hline PGG $1-3, n(\%)$ & $8(32)$ & $1(20)$ & 1.000 \\
\hline PGG $4-5, n(\%)$ & $17(68)$ & $4(80)$ & \\
\hline ASA $1-2, n(\%)$ & $23(92)$ & $4(80)$ & 0.473 \\
\hline ASA $3, n(\%)$ & $2(8)$ & $1(20)$ & \\
\hline CDS $0-2, n(\%)$ & $24(96)$ & $4(80)$ & 0.310 \\
\hline CDS $3 a-3 b, n(\%)$ & $1(4)$ & $1(20)$ & \\
\hline No RAD, $n(\%)$ & $24(96)$ & $5(100)$ & 1.000 \\
\hline $\mathrm{RAD}, n(\%)$ & $1(4)$ & $0(0.0)$ & \\
\hline
\end{tabular}

See also Table 1; LNP, number of positive nodes; $\left(^{*}\right)$, OR 2.871 (95\% CI 1,158-7,119; $p=0.023)$ and area under the curve (AUC) $=0.948(95 \%$ CI $0.851-1,000 ; p=0.002)$.

Marcilles's Node Involvement in Patients Undergoing RARP and ePLND for PCa
PCa patients undergoing open radical prostatectomy [9].

According to these considerations, Maderthaner et al. [9] have evaluated the effect of a new extended PLND template including the common iliac vessels and the Marcille's fossa compared to their previous template. In their study, patients with $\mathrm{pN}+$ disease had positive common iliac and Marcille's fossa LNs 17 and 7\%, respectively, without significantly increasing the risk of complications. Moreover, Marcille's node involvement was associated with metastases in other template location. Although the Authors performed multivariable logistic regression analysis of variables associated with LN metastases, no data were presented in order to evaluate risk factors associated with Marcille's nodal involvement [9].

In our study, $17 \%$ (5 out of 30 ) of $\mathrm{pN}+$ patients showed metastases on this area. Furthermore, analysing separately baseline and pathologic characteristics of those patients, it was found that high risk disease, familiarity, and contemporarily multiple LN metastases involvement in other template location were common characteristics. Considering the small sample on patients presenting lymph node involvement in Marcille's fossa, to perform a multivariable analysis was not possible. Moreover, we previously reported our analysis on the clinical predictors of LN invasion in low-, intermediate- and high-risk PCa patients who underwent ePLND $[17,34]$.

Urol Int 2019;103:25-32 
Table 3. Clinical and pathological characteristics of patients presenting with Marcille's nodes involvement

\begin{tabular}{|c|c|c|c|c|c|}
\hline & Patient 1 & Patient 2 & Patient 3 & Patient 4 & Patients 5 \\
\hline Age, year & 68 & 66 & 62 & 69 & 70 \\
\hline $\mathrm{BMI}, \mathrm{Kg} / \mathrm{m}^{2}$ & 23.5 & 33.1 & 24.8 & 30.9 & 22.4 \\
\hline Prostate volume, $\mathrm{mL}$ & 38 & 70 & 48.6 & 33.1 & 58 \\
\hline PSA & 4.7 & 8.0 & 6.8 & 12.4 & 20.5 \\
\hline Familiarity & Yes & Yes & No & Yes & Yes \\
\hline Biopsy \% core involvment & 56 & 70 & 83 & 67 & 31 \\
\hline Bioptic gleaso grouping, $n(\%)$ & 4 & 1 & 4 & 5 & 4 \\
\hline cT & 2 & 1 & 3 & 2 & 1 \\
\hline $\mathrm{cN}$ & 0 & 0 & 0 & 0 & 1 \\
\hline $\mathrm{cM}$ & 0 & 0 & 0 & 0 & 0 \\
\hline D' amico classification & High & Low & High & High & High \\
\hline Pathological gleason grouping, $n(\%)$ & 5 & 2 & 4 & 4 & 5 \\
\hline $\mathrm{pT}$ & $3 b$ & $2 c$ & $3 b$ & $3 b$ & $3 b$ \\
\hline \multicolumn{6}{|l|}{ Positve surgical margins } \\
\hline positive, $<1 \mathrm{~mm}$ & 0 & 0 & 1 & 0 & 0 \\
\hline positive, $\geq 1 \mathrm{~mm}$ & 0 & 1 & 0 & 0 & 1 \\
\hline $\mathrm{pN}$ & 1 & 1 & 1 & 1 & 1 \\
\hline Lymph node yielded, total & 33 & 27 & 41 & 45 & 30 \\
\hline Lymphnode yielded, right & 22 & 12 & 18 & 23 & 19 \\
\hline External iliac & 13 & 7 & 9 & 9 & 7 \\
\hline Obturator & 6 & 2 & 6 & 8 & 6 \\
\hline Cloquet & 1 & 1 & 1 & 1 & 1 \\
\hline Marcille & 2 & 2 & 2 & 5 & 2 \\
\hline Lymphnode yielded, left & 11 & 15 & 23 & 22 & 11 \\
\hline External iliac & 6 & 3 & 8 & 7 & 4 \\
\hline Obturator & 5 & 7 & 9 & 12 & 2 \\
\hline Cloquet & 0 & 1 & 1 & 1 & 2 \\
\hline Marcille & 0 & 2 & 6 & 2 & 2 \\
\hline Lymph nodes involved, total & 11 & 2 & 7 & 20 & 5 \\
\hline Lymph nodes involved, right & 9 & 2 & 1 & 1 & 2 \\
\hline External iliac & 5 & 0 & 1 & 1 & 1 \\
\hline Obturator & 2 & 1 & 0 & 0 & 1 \\
\hline Cloquet & 0 & 0 & 0 & 0 & 0 \\
\hline Marcille & 2 & 1 & 0 & 0 & 0 \\
\hline Lymph nodes involved, left & 2 & 0 & 6 & 19 & 3 \\
\hline External iliac & 2 & 0 & 2 & 7 & 0 \\
\hline Obturator & 0 & 0 & 1 & 9 & 1 \\
\hline Cloquet & 0 & 0 & 0 & 1 & 0 \\
\hline Marcille & 0 & 0 & 3 & 2 & 2 \\
\hline 90 days complications & 0 & 1 & $3 b$ & 0 & 0 \\
\hline
\end{tabular}

IQR, interquartile range; BMI, body mass index; PSA, prostate specific antigen; TPV, total prostate volume; BPC, biopsy positive cores; PW, prostate weight; LNR, lymph nodes removed; OT, operating time; DD, discharge day; $\mathrm{cT}$, tumour clinical stage; $\mathrm{cN}$, nodal; BGG, biopsy grade group; $\mathrm{pT}$, tumour pathological stage; NSM, negative surgical margin; PSM, positive surgical margin; PGG, pathological tumour grade group; ASA, American society of Anesthesiology score system; CDS, Clavien-Dindo score system; RAD, hospital re-admission.

Aggressiveness of tumour biology associates with multiple LN metastases. In our study, we have shown that Marcille's fossa are connected with the prostatic lymphatic system and their involvement associated with high metastatic load, which is supported by cancer biology. In this context, LNs of Marcille's fossa could represent a critical lymphatic landing sites connecting the pelvic and extra pelvic lymphatic system of the prostate.

To the best of our knowledge, this is the first study that analyses specifically the quantitative prevalence of Mar- 
cille's nodes and the incidence of malignancy involvement in a cohort of patients undergoing RARP and ePLND for PCa. Strengths of the paper are the prospective fashion; all the nodes packages were sent separately according to the most updated guidelines and were analysed and reviewed by a dedicated pathologist. However, the study is not without limitations, which include mainly different sets of patients collecting all risks group and the small number of patients with Marcille's node metastatic involvement. Wider multicentre series are needed in order to evaluate predictive factors of Marcille's nodes involvement in patients undergoing RARP and ePLND for PCa.

\section{Conclusion}

Marcille's nodes involvement is associated to contemporarily multiple LN metastases in other template locations in high-risk PCa patients. Wider series are required to comprehend predictor factors of Marcille's nodes involvement. Until then, the Marcille's lymphadenectomy, called "Marcillectomy", would be recommended when planning an ePLND in high-risk PCa patients and it should not be considered an option.

\section{Statement of Ethics}

We declare that all subjects have given their written informed consent. All procedures performed in studies involving human participants were in accordance with the ethical standards of the institutional and/or national research committee and with the 1964 Helsinki declaration and its later amendments or comparable ethical standards.

\section{Disclosure Statement}

The authors declare that they have no conflicts of interest to disclose.

\section{Funding Source}

No funding source was associated with this study.

\section{Authors Contributions}

A.B.P., G.E.C., M.S. and A.T. were responsible for analysis, and manuscript writing. W.A., A.B.C., G.E.C., M.S., A.T., V.D.M., F.M., and M.B. contributed to project development. A.B.P., G.E.C., and W.A. were responsible for manuscript editing. R.R., P.C., T.P., M.P., A.T., N.D.L., N.A., and D.I. collected all the required data.

\section{References}

1 Gil-Vernet JM. Prostate cancer: anatomical and surgical considerations. Br J Urol. 1996 Aug;78(2):161-8.

2 Heck MM, Retz M, Bandur M, Souchay M, Vitzthum E, Weirich G, et al. Topography of lymph node metastases in prostate cancer patients undergoing radical prostatectomy and extended lymphadenectomy: results of a combined molecular and histopathologic mapping study. Eur Urol. 2014 Aug;66(2): 222-9.

3 Fossati N, Willemse PM, Van den Broeck $\mathrm{T}$, van den Bergh RC, Yuan CY, Briers E, et al. The benefits and harms of different extents of lymph node dissection during radical prostatectomy for prostate cancer: a systematic review. Eur Urol. 2017 Jul;72(1): 84-109.

4 Briganti A, Blute ML, Eastham JH, Graefen M, Heidenreich A, Karnes JR, et al. Pelvic lymph node dissection in prostate cancer. Eur Urol. 2009 Jun;55(6):1251-65.

5 Mottet N, Bellmunt J, Bolla M, Briers E, Cumberbatch MG, De Santis M, et al. EAU-ESTRO-SIOG guidelines on prostate cancer. Part 1: screening, diagnosis, and local treatment with curative intent. Eur Urol. 2017 Apr;71(4):618-29.
6 Hueting TA, Cornel EB, Somford DM, Jansen $H$, van Basten JPA Pleijhuis RG, et al. External Validation of Models Predicting the Probability of Lymph Node Involvement in Prostate Cancer Patients. Eur Urol Oncol. 2018 Oct; 1(5)411-7.

7 Kim KH, Lim SK, Koo KC, Han WK, Hong SJ, Rha KH. Extended lymph node dissection in robot-assisted radical prostatectomy: lymph node yield and distribution of metastases. Asian J Androl. 2014 Nov-Dec;16(6): 824-8.

8 Yuh BE, Ruel NH, Mejia R, Wilson CM, Wilson TG. Robotic extended pelvic lymphadenectomy for intermediate- and high-risk prostate cancer. Eur Urol. 2012 May;61(5): 1004-10.

9 Maderthaner L, Furrer MA, Studer UE, Burkhard FC, Thalmann GN, Nguyen DP. More extended lymph node dissection template at radical prostatectomy detects metastases in the common iliac region and in the fossa of Marcille. BJU Int. 2018 May;121(5): 725-31.

10 Ventimiglia E, Briganti A, Montorsi F. Lymph node dissection during radical prostatectomy for prostate cancer: extending the template in the right patients without in- creasing complications. BJU Int. 2018 May; 121(5):677-8.

11 Lee P, Francis KE, Solomon MJ, RamseyStewart G, Austin KK, Koh C. Triangle of Marcille: the anatomical gateway to lateral pelvic exenteration. ANZ J Surg. 2017 Jul; 87(7-8):582-6.

12 Marcille M. Lymphatiques et ganglions iliopelviens. Université de Paris; 1902.

13 Dindo D, Demartines N, Clavien PA. Classification of surgical complications: a new proposal with evaluation in a cohort of $6336 \mathrm{pa}$ tients and results of a survey. Ann Surg. 2004 Aug;240(2):205-13.

14 D'Amico AV, Whittington R, Malkowicz SB, Schultz D, Blank K, Broderick GA, et al. Biochemical outcome after radical prostatectomy, external beam radiation therapy, or interstitial radiation therapy for clinically localized prostate cancer. JAMA. 1998 Sep;280(11): 969-74.

15 Briganti A, Larcher A, Abdollah F, Capitanio U, Gallina A, Suardi N, et al. Updated nomogram predicting lymph node invasion in patients with prostate cancer undergoing extended pelvic lymph node dissection: the essential importance of percentage of positive cores. Eur Urol. 2012 Mar;61(3):480-7. 
16 Porcaro AB, de Luyk N, Corsi P, Sebben M, Tafuri A, Mattevi D, et al.; Clinical Factors Predicting and Stratifying the Risk of Lymph Node Invasion in Localized Prostate Cancer. Clinical Factors Predicting and Stratifying the Risk of Lymph Node Invasion in Localized Prostate Cancer. Urol Int. 2017;99(2): 207-14.

17 Porcaro AB, de Luyk N, Corsi P, Sebben M, Tafuri A, Tamanini I, et al. Bilateral lymph node micrometastases and seminal vesicle invasion associated with same clinical predictors in localized prostate cancer. Tumori. 2017 May;103(3):299-306.

18 Porcaro AB, Siracusano S, de Luyk N, Corsi P, Sebben M, Tafuri A, et al. Low-Risk Prostate Cancer and Tumor Upgrading in the Surgical Specimen: Analysis of Clinical Factors Predicting Tumor Upgrading in a Contemporary Series of Patients Who were Evaluated According to the Modified Gleason Score Grading System. Curr Urol. 2017 Aug;10(3):11825.

19 Porcaro AB, Inverardi D, Corsi P, Sebben M, Cacciamani G, Tafuri A, et al. Prostate specific antigen levels and proportion of biopsy positive cores are independent predictors of upgrading patterns in low risk prostate cancer. Minerva Urol Nefrol. 2018, Epub ahead of print.

20 Srigley JR, Humphrey PA, Amin MB, Chang SS, Egevad L, Epstein JI, et al.; Members of the Cancer Committee, College of American Pathologists. Protocol for the examination of specimens from patients with carcinoma of the prostate gland. Arch Pathol Lab Med. 2009 Oct;133(10):1568-76.
21 Epstein JI, Egevad L, Amin MB, Delahunt B, Srigley JR, Humphrey PA; Grading Committee. The 2014 International Society of Urological Pathology (ISUP) consensus conference on Gleason grading of prostatic carcinoma: definition of grading patterns and proposal for a new grading system. Am J Surg Pathol. 2016 Feb;40(2):244-52.

22 Pini G, Matin SF, Suardi N, Desai M, Gill I, Porter J, et al. Robot assisted lymphadenectomy in urology: pelvic, retroperitoneal and inguinal. Minerva Urol Nefrol. 2017 Feb; 69(1):38-55.

23 Cacciamani GE, Porcaro AB, Sebben M, Tafuri A, Rizzetto R, De Luyk N, et al. Extended pelvic lymphadenectomy for prostate cancer: should the cloquet's nodes dissection be considered only an option? Minerva Urol Nefrol. 2019, Epub ahead of print.

24 Joniau S, Van den Bergh L, Lerut E, Deroose CM, Haustermans K, Oyen R, et al. Mapping of pelvic lymph node metastases in prostate cancer. Eur Urol. 2013 Mar;63(3): 450-8.

25 Eden CG, Arora A, Rouse P. Extended vs standard pelvic lymphadenectomy during laparoscopic radical prostatectomy for intermediate- and high-risk prostate cancer. BJU Int. 2010 Aug;106(4):537-42.

26 Briganti A, Chun FK, Salonia A, Gallina A, Farina E, Da Pozzo LF, et al. Validation of a nomogram predicting the probability of lymph node invasion based on the extent of pelvic lymphadenectomy in patients with clinically localized prostate cancer. BJU Int. 2006 Oct;98(4):788-93.

27 Heidenreich A, Ohlmann $\mathrm{CH}$, Polyakov S. Anatomical extent of pelvic lymphadenectomy in patients undergoing radical prostatectomy. Eur Urol. 2007 Jul;52(1):29-37.
28 Ramírez-Backhaus M, Mira Moreno A, Gómez Ferrer A, Calatrava Fons A, Casanova J, Solsona Narbón E, et al. Indocyanine Green Guided Pelvic Lymph Node Dissection: An Efficient Technique to Classify the Lymph Node Status of Patients with Prostate Cancer Who Underwent Radical Prostatectomy. J Urol. 2016 Nov;196(5):1429-35.

29 Nguyen DP, Huber PM, Metzger TA, Genitsch V, Schudel HH, Thalmann GN. A Specific Mapping Study Using Fluorescence Sentinel Lymph Node Detection in Patients with Intermediate- and High-risk Prostate Cancer Undergoing Extended Pelvic Lymph Node Dissection. Eur Urol. 2016 Nov;70(5): 734-7.

30 Osmonov DK, Boller A, Aksenov A, Naumann $\mathrm{M}$, Jünemann KP. [Intermediate and high risk prostate cancer patients. Clinical significance of extended lymphadenectomy]. Urologe A. 2013 Feb;52(2):240-5.

31 Zehnder P, Desai M. Extended lymph node dissection: bladder, kidney. Curr Opin Urol. 2011 Mar;21(2):110-4.

32 Kitamura $\mathrm{H}$, Masumori N, Tsukamoto $\mathrm{T}$. Role of lymph node dissection in management of bladder cancer. Int J Clin Oncol. 2011 Jun;16(3):179-85.

33 Roth B, Burkhard FC. The Role of Lymphadenectomy in Radical Cystectomy. Eur Urol Suppl. 2010;9(1):19-24.

34 Porcaro AB, De Luyk N, Corsi P, Sebben M, Tafuri A, Processali T, et al. Clinical factors predicting bilateral lymph node invasion in high-risk prostate cancer. Urol Int. 2017; 99(4):392-9. 